\section{Cahiers de Narratologie}

Analyse et théorie narratives

$20 \mid 2011$

Voix off et narration cinématographique

\title{
Relativisme cognitif et indétermination sémiotique : abduction et méta-abduction dans l'œuvre romanesque d'Umberto Eco
}

Ilias Yocaris

\section{OpenEdition}

Journals

Édition électronique

URL : http://journals.openedition.org/narratologie/6385

DOI : 10.4000/narratologie.6385

ISSN : 1765-307X

Éditeur

LIRCES

Référence électronique

Ilias Yocaris, "Relativisme cognitif et indétermination sémiotique : abduction et méta-abduction dans l'œuvre romanesque d'Umberto Eco », Cahiers de Narratologie [En ligne], 20 | 2011, mis en ligne le 11

février 2016, consulté le 19 avril 2019. URL : http://journals.openedition.org/narratologie/6385 ; DOI

$10.4000 /$ narratologie.6385

Ce document a été généré automatiquement le 19 avril 2019

Article L.111-1 du Code de la propriété intellectuelle. 


\title{
Relativisme cognitif et indétermination sémiotique : abduction et méta-abduction dans l'œuvre romanesque d'Umberto Eco
}

\author{
Ilias Yocaris
}

\section{Introduction : le problème de la compréhension}

1 L'œuvre romanesque d'Umberto Eco peut être considérée à plusieurs égards comme un traité de sémiotique «fictionnalisé " en plusieurs volumes, qui vient compléter les ouvrages théoriques de l'auteur (on pense surtout à trois livres : Opera aperta, Trattato di semiotica generale et I Limiti dell'interpretazione). Or, la question principale posée par ce «traité » sui generis porte justement sur le problème de la compréhension: pour Eco comme pour ses héros fictionnels, «le monde est une énigme» (PF 100) qu'il s'agit de déchiffrer, afin de reconstituer si possible un ordre « qui le fasse sortir de son effroyable confusion" (PF 222). La quête de cet ordre est systématiquement mise en avant dans les quatre romans qui formeront notre corpus (Il Nome della rosa, Il Pendolo di Foucault, L'Isola del giorno prima, Baudolino). En effet, les protagonistes respectifs de ces romans se retrouvent invariablement confrontés à un ensemble d'enchaînements factuels énigmatiques perçus comme autant de signes à partir desquels ils essaient de comprendre le mode de fonctionnement du monde qui les entoure et d'en (re)constituer une représentation mentale cohérente. Comme l'auteur le souligne lui-même dans I Limiti dellinterpretazione, une telle démarche herméneutico-épistémologique relève pour l'essentiel de deux opérations sémiotiques : (a) l'abduction ; (b) la méta-abduction.

2 (a) L'abduction. L'abduction peut être décrite de manière synthétique comme « la reductio ad unum d'une pluralité » (Eco 1992 : 262) : confronté à une série décousue de faits $(x, y, z$ ...) apparemment inexplicables, l'observateur tente de trouver une interprétation commune qui permette de rendre intelligible l'enchaînement de ces faits, en les intégrant 
dans " une séquence cohérente» (Eco 1992: 272). Le processus abductif se présente en somme comme un «jeu d'assemblage » (PF 125) d'éléments a priori épars, entre lesquels il s'agit de faire émerger un ensemble de connexions permettant de dégager un schéma organisationnel d'ensemble (cf. figure 1).

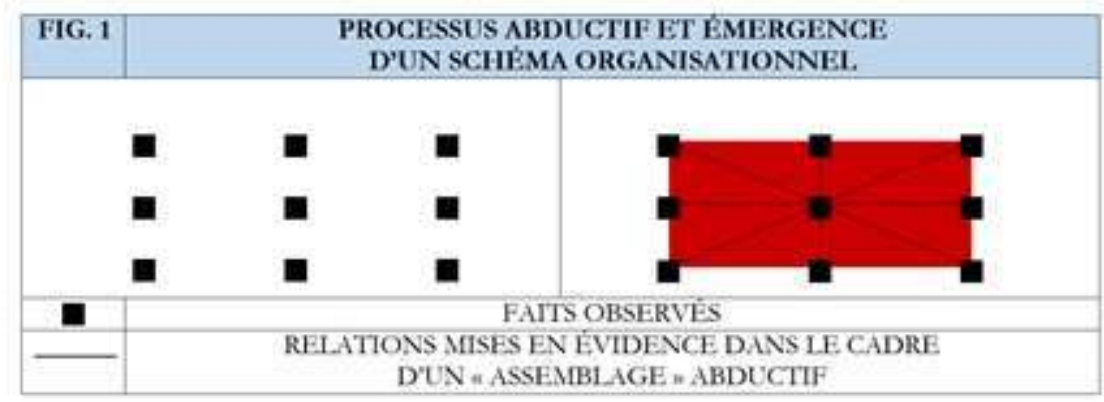

L'exemple parfait de ce type de démarche est fourni par l'enquête policière menée par Guillaume de Baskerville dans Il Nome della Rosa (cf. Yocaris 2006a). En effet, Guillaume doit se pencher sur une série de données factuelles apparemment sans lien entre elles que l'on peut sommairement résumer comme suit : (1) dans l'abbaye où il se trouve, plusieurs moines sont retrouvés morts, les uns après les autres ; (2) tous ces moines ont eu en leur possession, à un moment donné, un livre mystérieux, qui s'avérera être le volume perdu de la Poétique d'Aristote consacré à la comédie ; (3) certains des cadavres ont des taches d'encre sur les doigts et la langue. Après mûre réflexion, Guillaume construit un schéma abductif qui permet de mettre en évidence les liens qui existent entre (1), (2) et (3), en faisant émerger une "version des faits " cohérente: les pages de l'ouvrage ont été enduites de poison par quelqu'un qui ne veut pas que le contenu du livre soit divulgué ; de la sorte, ceux qui les tournent en s'humectant les doigts sont empoisonnés au fur et à mesure que progresse leur lecture ...

4 (b) La méta-abduction. La méta-abduction est définie par Eco comme la confrontation du «monde possible» construit par l'observateur avec le «monde actuel» dans lequel il évolue, afin de décider «si l'univers possible déterminé par [ses] abductions de premier niveau est identique à l'univers de [son] expérience » (Eco 1992 : 264). Ainsi, dans Il Nome della Rosa, Guillaume de Baskerville finit par retrouver l'assassin mystérieux qu'il traquait depuis le début, Jorge de Burgos, et ce dernier confirme la validité de son schéma abductif en admettant qu'il a bel et bien empoisonné le livre d'Aristote.

5 Le processus (méta-)abductif permet donc en substance de rationaliser le réel, en dégageant une série de principes explicatifs susceptibles de donner au monde un sens, de lier entre eux les phénomènes (au sens le plus large de ce mot) observés par l'homme dans une infinité de situations et de cadres cognitifs différents : une « reconstitution des faits » dans le cadre d'une enquête policière, un diagnostic médical, un éditorial politique, une théorie scientifique constituent autant d'assemblages abductifs permettant a priori de " découvrir et [de] révéler des connexions entre les choses » (IGP 115). Une telle démarche ne saurait être assimilée simplement à une description approfondie des "objets" considérés (les différentes suites de phénomènes sous-tendus par le même principe explicatif), dans la mesure où l'on s'attache en réalité à formaliser ces « objets » : ainsi par exemple, dans Baudolino, le débat théorique entre Boron et Ardzrouni sur l'existence du vide (cf. B 387-389, 421-425) laisse entr'apercevoir deux univers aux caractéristiques diamétralement opposées... On notera donc que toute abduction est une construction 
interprétative transformant le regard a priori neutre de l'observateur en un "voir comme ", au sens wittgensteinien du terme (cf. Investigations philosophiques, II, 11).

Or les opérations intellectuelles liées à la mise en place d'un agencement (méta-)abductif ne vont pas sans soulever toute une série de questionnements philosophiques qui se font jour dans les romans de notre corpus: pour aller vite, Eco considère ces opérations comme un vrai pari épistémologique que l'observateur n'a aucune certitude de gagner, dans la mesure où il est confronté à une réalité évanescente, intrinsèquement ambiguë et complexe qui n'est pas forcément réductible à un schéma explicatif totalisant et univoque (cf. Eco $1962:$ 2). Ce scepticisme méthodologique, typiquement postmoderne, est mis en évidence à travers les références ironiques au roman policier du $19^{\text {ème }}$ siècle (et notamment la série des Sherlock Holmes) qui reviennent sans cesse dans Il Nome della rosa. Quelle est la signification de ces références? Les pionniers du whodunit (E. A. Poe et A. Conan Doyle), fortement imprégnés par les schémas de pensée inspirés ou hérités du rationalisme positiviste (cf. Tani 1984:11, 17-18 et passim), développent en réalité dans leurs ouvrages fictionnels toute une réflexion sémiotico-épistémologique sur le processus (méta-)abductif, en tentant de montrer qu'il existe certains pré-requis conceptuels censés garantir la pertinence des schémas explicatifs élaborés par la figure du détective en toute circonstance $^{1}$. Comme nous l'avons montré ailleurs (v. Yocaris 2006a: 201-202), ces prérequis sont au nombre de trois : (1) il existe un cloisonnement strict entre " observant " et "observé ", en l'occurrence entre la figure de l'enquêteur et les enchaînements factuels qu'il tente de (qu'il finira nécessairement par) mettre au jour ; (2) la structure du « réel » est en tous points conforme à nos représentations mentales; (3) il existe un rapport déterministe et univoque entre chaque signe et l'« objet » auquel il renvoie. Or, dans les romans de notre corpus, ces trois postulats sont fortement remis en question : si Eco choisit de tourner en dérision le "récit de ratiocination " traditionnel, c'est qu'il a conscience de vivre dans un monde en partie indéchiffrable, où les observateurs sont confrontés à une suite discontinue de phénomènes dont rien ne garantit la réalité objective, l'intelligibilité et la transparence sémiotique.

\section{La fin de l'« objectivité »: les limites du dualisme philosophique}

7 La tâche des enquêteurs qui interviennent dans les romans policiers "classiques » est grandement facilitée du fait que le schéma abductif qui leur sert de fil conducteur n'a en général aucune incidence sur le déroulement de l'affaire qu'ils se proposent d'élucider : considéré sous un angle purement philosophique, le whodunit traditionnel repose en fait sur une forme de «dualisme" (le fameux " partage cartésien » entre res cogitans et res extensa), puisqu'il présuppose dans la plupart des cas la présence d'un cloisonnement assez strict entre "l'instance observatrice» (le détective) et l'« objet observé » (l'enchaînement factuel que ce dernier tente de mettre au jour). C'est avant tout la croyance sous-jacente à la validité universelle des schémas épistémologiques dualistes qui confère à des détectives comme Sherlock Holmes, Rouletabille ou Hercule Poirot la certitude d'être "dans le vrai », de comprendre «ce qui s'est réellement passé ». Or, le moins que l'on puisse dire, c'est que cette croyance s'effrite dangereusement dans les romans de notre corpus. Pourquoi? En termes épistémologiques, on dirait que les personnages de Eco se trouvent confrontés à deux problèmes majeurs : (a) l'émergence 
d'interactions récursives entre «observant» et «observé»; (b) l'émergence d'enchaînements phénoménaux descriptibles comme des « objets ambigus ».

8 (a) Les interactions récursives entre "observant » et "observé». Les protagonistes des romans du corpus sont déstabilisés du fait que les « mondes possibles » qu'ils élaborent quand ils mettent en place un assemblage abductif influent rétroactivement sur le « monde actuel » au sein duquel ils évoluent, en modifiant le cours même des « événements » qu'ils tentent d'expliquer : comme le dit Baudolino, «à imaginer d'autres mondes, on finit par changer aussi celui-ci » $\left(B_{\left.130^{2}\right)}\right.$. Ainsi par exemple, dans Il Nome della rosa, Guillaume postule à tort que l'instigateur des meurtres sur lesquels il enquête (Jorge de Burgos) suit « le rythme des sept trompettes de l'Apocalypse » (NR 475 ; cf. NR 310-311, 371 etc.), mais ce dernier finit par se conformer « réellement » à ce schéma abductif pour le leurrer (cf. Yocaris 2006a : 202-203).

Le dualisme philosophique se trouve donc en l'occurrence aboli parce que les schémas abductifs mis en place par les différents personnages fonctionnent comme des "prophéties autoréalisatrices » : c'est parce que Casaubon, Belbo et Diotallevi postulent l'existence d'un complot universel ourdi par les Templiers et les Rose-Croix pour récupérer une carte imaginaire que ces derniers se mettent réellement à les poursuivre pour s'approprier la carte, dont l'existence ne fait plus aucun doute pour eux. Un roman comme Il Pendolo di Foucault repose ainsi dans sa totalité sur une "boucle récursive " puisque l'effet rétroagit sur la cause qui l'a engendré, d'où telle mise en abyme qui apparait dès le premier chapitre, la description du chœur de l'église de Saint-Martin-desChamps :

(1) J'ai cherché alors à distraire mon regard en suivant la courbe qui, partant des chapiteaux des colonnes disposées en demi-cercle, se dirigeait le long des nervures de la voûte vers la clef, répétant le mystère de l'ogive, qui se soutient sur une absence, suprême hypocrisie statique, et fait croire aux colonnes qu'elles poussent vers le haut les liernes, et à celles-ci, repoussées par la clef, qu'elles fixent à terre les colonnes, la voûte étant en revanche un tout et un rien, effet et cause en même temps. (PF 11 ; je souligne)

La situation se trouve encore aggravée du fait que les constructions interprétatives élaborées par les héros d'Eco informent leur vision du monde par autosuggestion: le cloisonnement entre observant et observé est aussi mis à mal parce que les personnages placés en position d'« observateur» ont tendance à prendre leurs désirs/leurs peurs/ leurs obsessions personnelles pour des réalités "objectives", comme on le voit par exemple en (2):

(2) Cette nuit-là, il [Roberto, qui est obsédé par l'image d'une colombe orangée] était sur le point de décider qu'il serait resté tous les jours à venir sur le vaisseau. Mais en levant les yeux au ciel, il avait vu un groupe d'étoiles qui, d'un coup, parurent lui montrer le profil d'une colombe aux ailes tendues, laquelle portait dans sa bouche un rameau d'olivier. Or il est bien vrai que dans le ciel austral, à une courte distance du Grand Chien, on avait déjà repéré depuis au moins quarante ans une constellation de la Colombe. Mais je ne suis pas du tout sûr que Roberto, d'où il se trouvait, à cette heure et en cette saison, eût pu précisément apercevoir ces étoiles. En tous les cas, puisque ceux qui y avaient vu une colombe [...] montraient encore plus d'imagination que n'en avait Roberto, je dirais que n'importe quelle disposition d'astres, à ce moment-là, pouvait apparaître à Roberto comme un pigeon, un biset, un ramier ou palombe, une tourterelle, ce que vous voulez: bien que, au matin, il eût douté de son existence, la Colombe Couleur Orange s'était fixée dans sa tête comme un clou [...]. (IGP, 342 ; cf. PF 46, IGP 101, 225 et passim). 
11 (b) Les « objets ambigus ». La remise en cause du dualisme philosophique dans les romans $\mathrm{du}$ corpus se manifeste également par l'émergence d'enchaînements phénoménaux descriptibles comme des « objets ambigus » : qu'est-ce à dire ? Un « objet ambigu » est une entité possédant des caractéristiques intrinsèquement contradictoires, dans la mesure où sa configuration globale dépend constitutivement de la perspective observationnelle que l'on projette sur elle : il en est ainsi par exemple des fameux « cubes de Necker ", dont les parois saillent et s'enfoncent en même temps selon la manière dont on choisit de les regarder (cf. fig. 2).

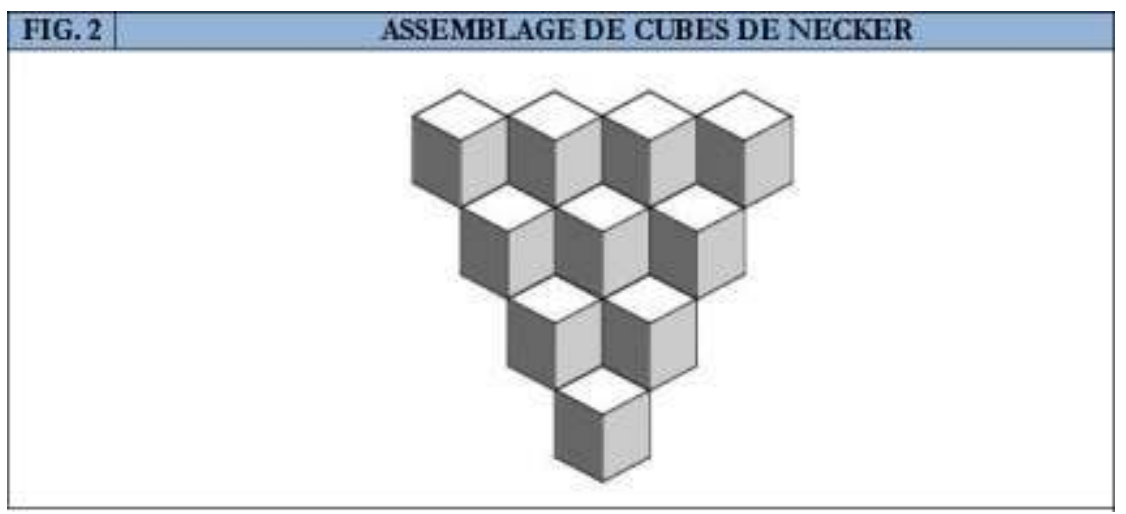

Or, dans certains cas de figure, les personnages mis en scène par Eco doivent analyser des situations si complexes qu'ils font successivement ressortir deux agencements abductifs mutuellement exclusifs qui se superposent, formant ainsi un « objet ambigu» (cf. fig. 3). Ainsi par exemple, dans Il Pendolo di Foucault, Casaubon finit par comprendre que l'affrontement ultime entre Aglié et Belbo, qui aboutira à la mort de ce dernier, est susceptible de deux interprétations contradictoires (cf. PF 603, 628) : (1) Agliè fait tuer Belbo parce qu'il a refusé de révéler son secret, à savoir l'emplacement exact de l'Umbilicus Telluris, le Centre du Monde (cf. PF 459-468) ; (2) Agliè fait tuer Belbo pour qu'il ne révèle pas ce secret, le privant du même coup de « la vague aura qui lui conférait charisme et pouvoir » (PF 628). Certes, ces deux interprétations ne sont pas placées tout à fait sur le même plan, dans la mesure où la deuxième semble à Casaubon plus plausible que la première (cf. PF 627-628) ; il n'en reste pas moins que leur juxtaposition entraîne une "anamorphose » assez radicale de l'« objet» de référence (l'enchaînement événementiel qui a abouti à la mort de Belbo), ébranlant ainsi la confiance du lecteur - et de Casaubon lui-même - aux schémas épistémologiques intégralement dualistes. 


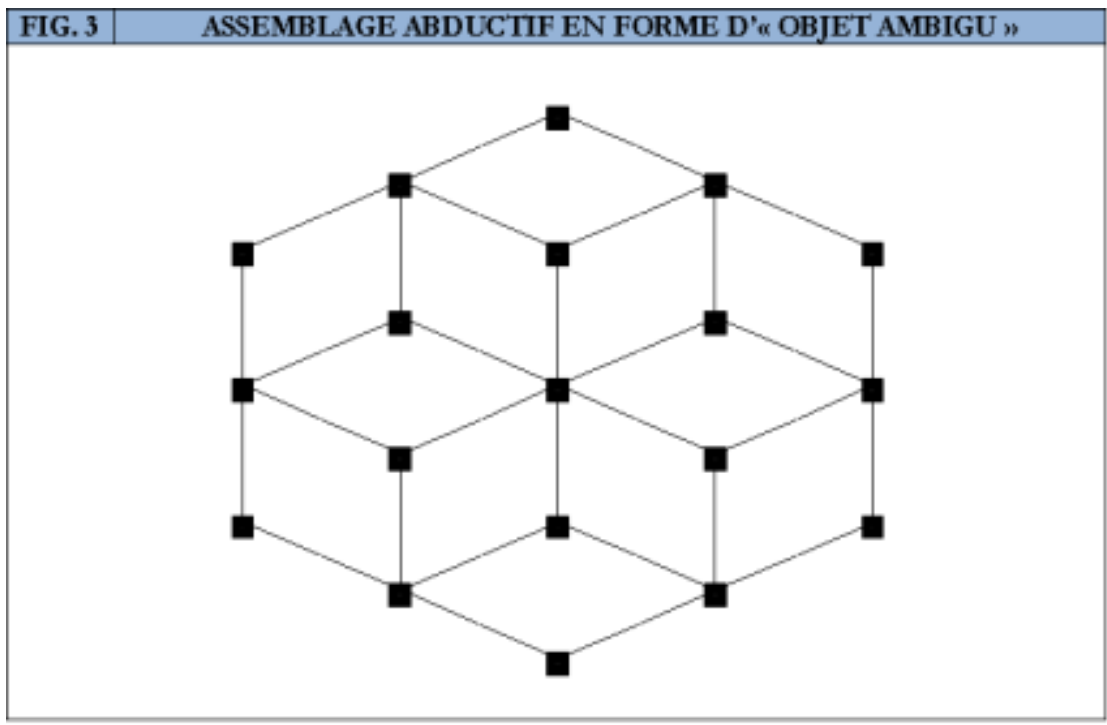

13 L'importance cruciale accordée par Eco (à l'instar de tous les auteurs postmodernes) au problème des « objets ambigus» est du reste confirmée dans Il Pendolo di Foucault par la présence d'une mise en abyme, Casaubon décrivant les jardins d'un château où il a décidé de se rendre à la demande d'Agliè pour assister à une cérémonie occultiste :

(3) Nous montions, et de terrasse en terrasse les jardins changeaient de physionomie. Certains avaient forme de labyrinthe, d'autres figure d'emblème, mais on ne pouvait voir le dessin des terrasses inférieures que des terrasses supérieures, si bien que j'aperçus d'en haut le contour d'une couronne et beaucoup d'autres symétries que je n'avais pas pu remarquer quand je les parcourais, et qu'en tout cas je ne savais pas déchiffrer. Chaque terrasse, pour qui se déplaçait au milieu des haies, par effet de perspective offrait certaines images mais, revue de la terrasse supérieure, procurait de nouvelles révélations, et même de sens opposé - et chaque degré de cette échelle parlait deux langues différentes au même moment. (PF 344)

L'homologie entre la description des terrasses du château et les développements théoriques consacrés par Eco au processus abductif est évidente: chaque terrasse est l'équivalent d'une suite de phénomènes susceptible de donner lieu à plusieurs "lectures ", à plusieurs assemblages abductifs différents (voire contradictoires) en même temps, en fonction du point de vue «herméneutique » adopté par l'observateur. Voilà pourquoi Agliè insiste par la suite sur le fait que chaque jardin se présente en définitive comme une opera aperta, puisqu'il est « lisible comme un livre » (ibid.).

\section{La mort de Dieu : I'avènement du faillibilisme épistémologique}

L'incertitude pesant sur la démarche (méta-)abductive est encore accrue du fait que les héros d'Eco évoluent dans un monde où il n'existe plus aucune instance métaphysique susceptible de garantir en toutes circonstances l'adéquation entre leurs représentations mentales et la structure même du «réel ». Pour les détectives qui officient dans les "récits de ratiocination » du $19^{\text {ème }}$ siècle, cette adéquation va de soi, dans la mesure où leur démarche repose implicitement sur le postulat épistémologique de l'adequatio mentis ad rem : comme l'explique Eco dans I Limiti dell'interpretazione, les raisonnements pseudo-scientifiques élaborés par Dupin et Sherlock Holmes reposent en réalité sur un fondement purement métaphysique, à savoir l'idée (héritée de la philosophie cartésienne, 
spinozienne et leibnizienne) que Dieu "a sculpté en notre âme une faculté de pensée pouvant opérer en accord avec les lois de la nature " (Eco 1992: 281). Or, les héros des romans de notre corpus constatent tous à leurs dépens que le monde dans lequel ils évoluent s'avère dans certaines circonstances profondément différent de leurs représentations mentales: il suffit de penser à la mort par noyade de Caspar Wandendrossel, fermement persuadé qu'il pouvait marcher au fond de la mer abrité sous une cloche (cf. IGP 329-337)... Dès lors, pour les personnages d'Eco, deux conclusions s'imposent de facto: (a) Dieu n'existe pas; (b) les conjectures abductives sont par définition faillibles (cf. Cannon 1989 : 87-88). Le rapport très étroit qui existe entre (a) et (b) est mis en évidence :

- Dans Il Nome della rosa, du fait de l'échec inattendu de Guillaume de Baskerville : constatant que le «monde possible » qu'il a mentalement élaboré ne correspond pas à ce qui s'est « réellement » passé dans l'abbaye, ce deleuzien ante litteram aboutit à la conclusion qu' «il n'est point d'ordre dans l'univers » (NR 497), que ce dernier n'est donc pas réductible «à l'ordre que notre esprit imagine » (ibid.), et que Dieu ne saurait exister dans la confusion (d'où ses dernières paroles : « Non in commotione, non in commotione Dominus », NR 4983).

- Dans Il Pendolo di Foucault, du fait de la réussite inespérée de Belbo et de ses amis : constatant que le schéma abductif délibérément extravagant qu'ils ont élaboré (une conspiracy theory des plus délirantes) finit par informer performativement le « réel » fictionnel, ces derniers ironisent sur la crédulité des occultistes qui sont prêts à valider ce schéma sans aucune vérification préalable en affirmant qu'il est étayé par « une série de faits, vrais [...] comme Dieu est vrai » (PF 499).

\section{L'opacification du monde : la fin du déterminisme sémiotique}

Les héros des romans policiers "classiques » ont enfin un dernier avantage sur les personnages mis en scène par Eco : ils évoluent dans un monde où tout est transparent, un monde peuplé de signes fonctionnant comme des «étiquettes » qui renvoient chacune à un - et un seul - référent. Ainsi par exemple, quand le Rouletabille de Gaston Leroux lit par hasard (dans Le Mystère de la chambre jaune) une petite annonce signée M.A.T.H.S.N., il en déduit, "sans autre forme de procès ", que ces initiales doivent renvoyer forcément au NP «Mathilde Stangerson» (le nom de la femme agressée dans la "chambre jaune ») : Rouletabille exclut d'emblée toute autre possibilité, ce qui facilite grandement l'assemblage des pièces du "puzzle » abductif qu'il est en train de mettre en place. La présence d'un lien fixe et bi-univoque entre un signe-étiquette et l'« objet » auquel il se rattache a une importance philosophique décisive, dans la mesure où elle permet de créer un univers intégralement fondé sur l'Identité et la Récognition, un univers positiviste d'où l'altérité et l'indétermination sous toutes leurs formes sont définitivement bannies. Or, le déterminisme sémiotique professé par Holmes, Dupin et leurs épigones conceptuels est systématiquement tourné en dérision par Eco, dont les personnages se retrouvent égarés dans un vrai labyrinthe de connexions sémiotiques parfois impossibles à démêler. Qu'est-ce qui a occasionné ce basculement ? L'« opacification » du « réel » fictionnel, qui cesse d'être immédiatement «lisible» dans les romans de notre corpus, découle essentiellement d'une conjonction de trois facteurs: (a) l'existence de signes irréductiblement ambigus et polysémiques; (b) la prolifération incontrôlable des connexions «signifiantes »; (c) l'imbrication de «territoires » conceptuels hétéroclites, 
dans la mesure où chacun est indissociablement lié à des modes de pensée et des appariements sémiotiques différents.

(a) L'existence de signes irrémédiablement ambigus. L'élaboration de schémas (méta)abductifs repose principalement sur l'interprétation de certains signes qui s'offrent au regard de l'«observateur» (indices de toutes sortes, messages codés qui doivent être déchiffrés etc.). Dans les «récits de ratiocination" traditionnels, l'activité « herméneutique » de l'enquêteur se présente en général comme une découverte : chaque signe a un sens précis et univoque que l'on s'attache simplement à mettre au jour en utilisant une " méthode de travail » appropriée. Ainsi par exemple, dans "The Gold-Bug " («Le Scarabée d'or ») d'E. A. Poe, William Legrand parvient à déchiffrer sans peine un message codé a priori totalement incompréhensible [cf. (4a), (4b)] en posant une série de restrictions préalables qui lui permettent de réduire sans cesse le nombre d'interprétations possibles jusqu'à trouver la «bonne » - celle qui doit rester forcément à la fin, quand toutes les autres ont été éliminées ${ }^{4}$.
(4a) 53++!305))6*;4826)4+)4+);:806*;48!8]60))85;1+8*:+(;:+*8!83(88)5*!;46(;88*96*?;8)*
$\left.\left.+(; 485) ; 5^{*} ! 2:^{*}+\left(; 4956^{*} 2\left(5^{*}\left[{ }_{0} 4\right) 8\right] 8^{*} ; 4069285\right) ;\right) 6 ! 8\right) 4++;(+9 ; 48081 ; 8: 8+1 ; 48 ! 85 ; 4) 485 !$
$528806^{*} 81(+9 ; 48 ;(88 ; 4(+? 34 ; 48) 4+; 161 ;: 188 ;+?$;
(4b) A good glass in the bishop's hostel in the devil's seat forty-one degrees and thirteen minutes northeast and by north main branch seventh limb east side shoot from the left eye of the death's head a bee-line from the tree through the shot fifty feet out

Il n'en va pas de même pour les personnages mis en scène par Eco : pour eux, l'activité herméneutique, loin d'être une découverte, implique presque toujours une série de choix, puisqu'ils découvrent que chaque significans peut potentiellement renvoyer à plusieurs significata différents entre lesquels ils sont obligés de faire le tri en se fiant parfois à leur seule intuition. Ainsi, dans Il Pendolo di Foucault, le document tronqué que le colonel Ardenti montre à Belbo et ses compagnons [cf. (5a)] est susceptible de deux lectures diamétralement opposées : Ardenti y voit un message codé que les Templiers de Provins ont légué à la postérité [cf. (5b)], Lia, la compagne de Casaubon, le lit plus prosaïquement comme une « liste des commissions » (PF 543, cf. (5c)].

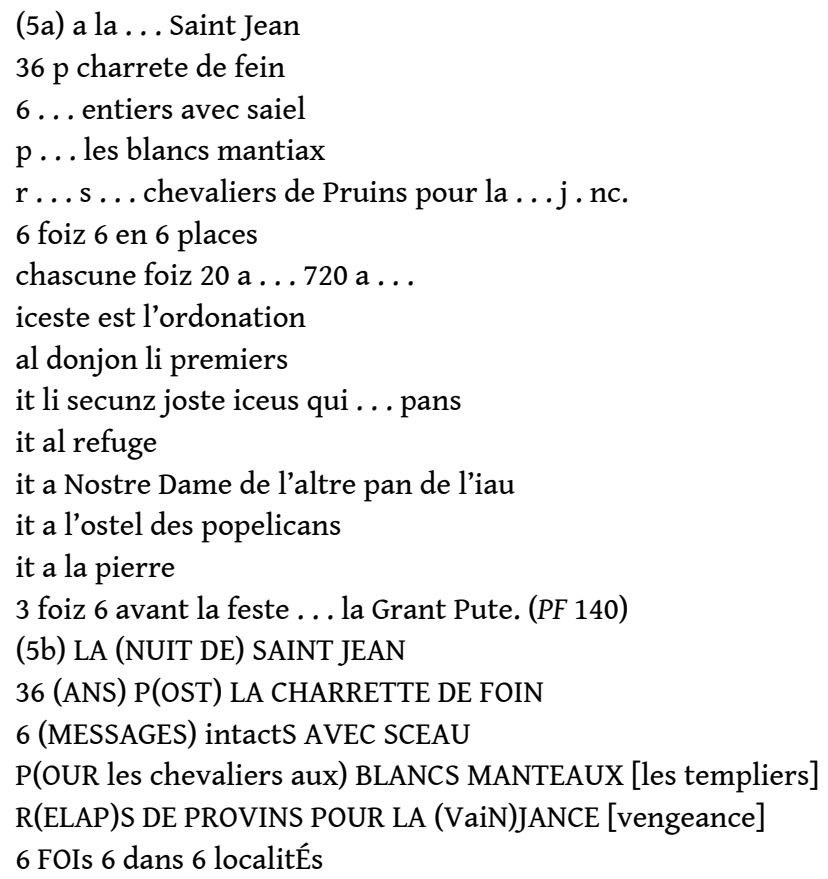




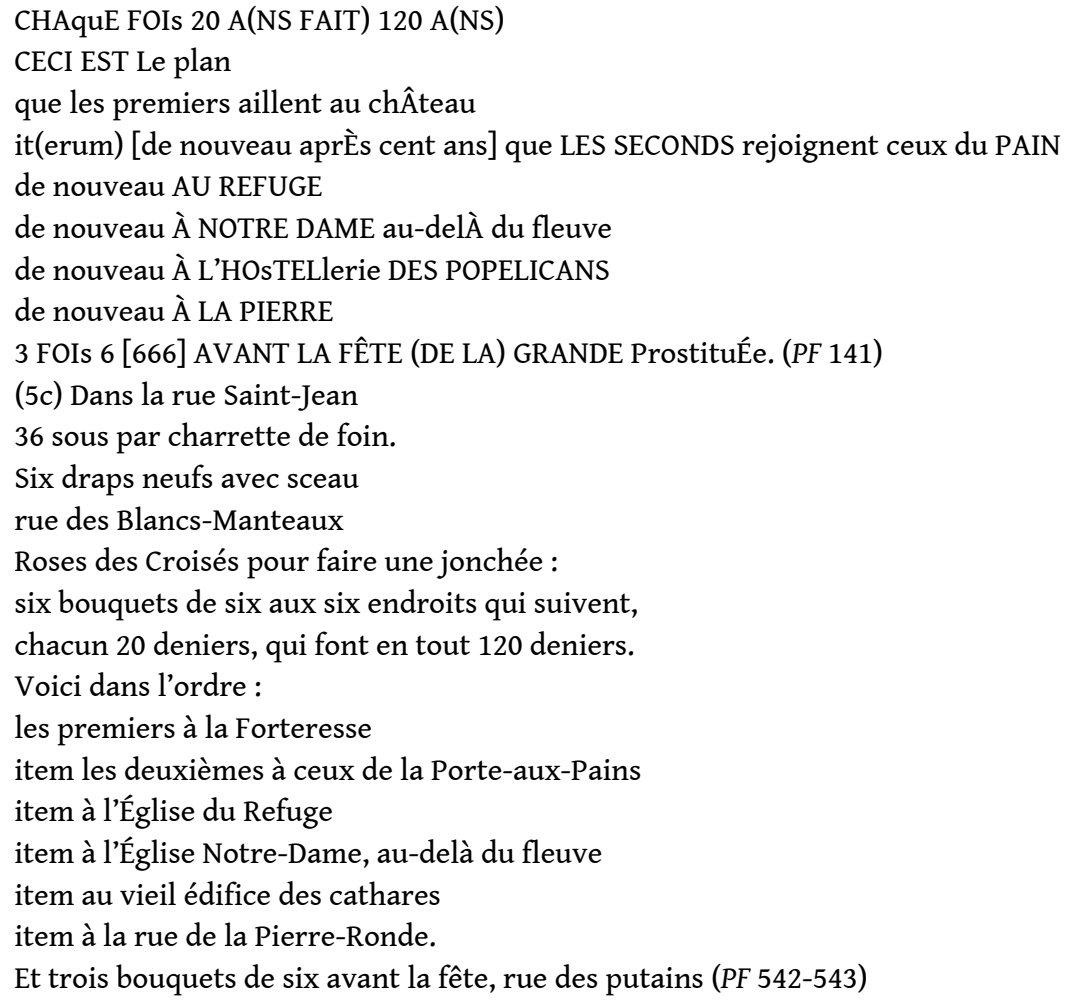

19 Même si la deuxième exégèse est infiniment plus plausible que la première, la simple ramification des possibles interprétatifs qui se fait jour en l'occurrence ébranle profondément les fondements du déterminisme sémiotique professé par Legrand: ce n'est pas un hasard si Belbo évoque ironiquement «Le Scarabée d'or » quand il est interrogé par le commissaire De Angelis sur son entretien avec Ardenti (cf. PF 172).

(b) La prolifération des connexions signifiantes. Obligés d'effectuer sans cesse des choix sémiotiques dont rien ne garantit la pertinence, les personnages mis en scène par Eco finissent par comprendre que chaque suite de phénomènes peut potentiellement donner lieu à une infinité de schémas abductifs différents, puisque la variété des relations que l'on peut établir entre les éléments d'un ensemble donné est pratiquement inépuisable : comme l'explique De Angelis à Casaubon dans son langage vernaculaire, « en ce monde tout a quelque chose à voir avec tout » (PF 320 ; cf. fig. 4). On en veut pour preuve, entre autres :

21 - La « fureur combinatoire» (PF 28) des héros du Pendule de Foucault, capables de dégager des schémas abductifs même à partir d'une série de données tout à fait incohérente : ainsi par exemple, Belbo construit à partir de (6) tout un schéma explicatif permettant de déduire ( ?) que « Jésus est le fondateur de la lignée royale de France » (PF 383)...

(6) Les Templiers y sont toujours pour quelque chose

Ce qui suit n'est pas vrai

Jésus a été crucifié sous Ponce Pilate

Le sage Ormus fonda en Égypte les Rose-Croix

Il y a des kabbalistes en Provence

Qui s'est marié aux noces de Cana?

Minnie est la fiancée de Mickey

Il en découle que

$\mathrm{Si}$

Les druides vénéraient les vierges noires

Alors 
Simon le Magicien identifie la Sophia à une prostituée de Tyr

Qui s'est marié aux noces de Cana?

Les Mérovingiens se disent rois de droit divin

Les Templiers y sont toujours pour quelque chose (PF 382)

- La ramification des possibles fictionnels dans les derniers chapitres de Baudolino, où l'on voit successivement émerger trois « versions des faits » concurrentes permettant de rattacher la mort de l'empereur Frédéric à des enchaînements factuels différents : (1) Frédéric a été tué par un des personnages accusés par le Poète (Boron, Kyot, Boïdi) ; (2) il a été empoisonné par le Poète lui-même ; (3) il est mort accidentellement (cf. B 629-639, 649-654). Même si (3) semble finalement l'emporter sur (1) et (2), l'absence de toute procédure de vérification méta-abductive ne permet pas au lecteur/aux protagonistes du roman de se former une opinion définitive sur ce point, contrairement à ce qui se passe dans Il Nome della rosa par exemple.

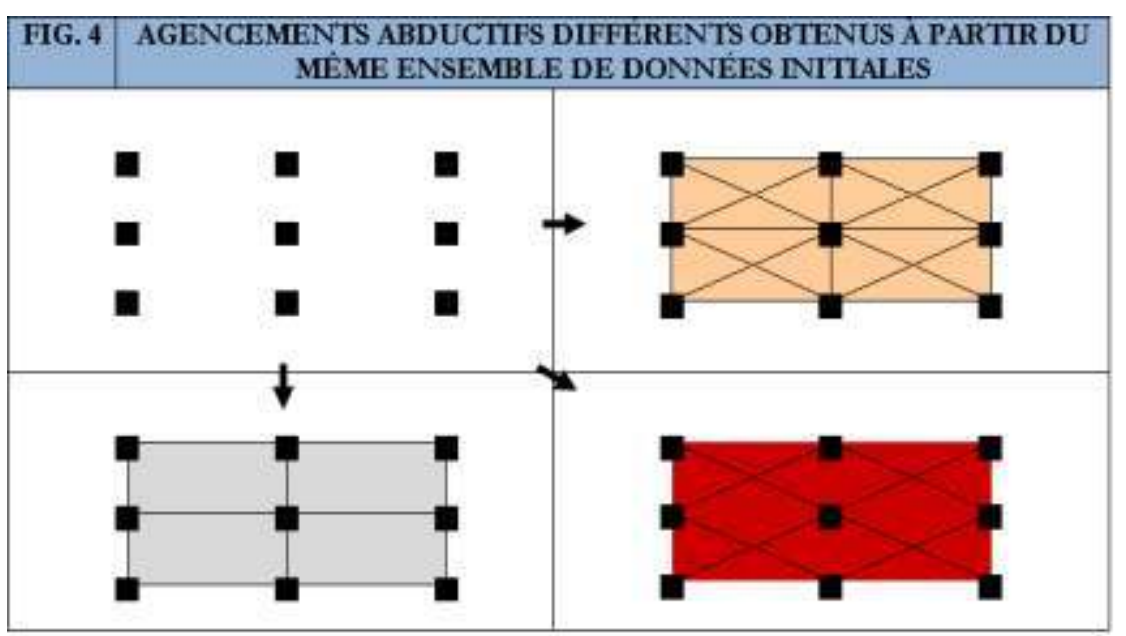

Cette multiplication ad infinitum des connexions signifiantes plonge les héros d'Eco dans un véritable vertige ontologique: confrontés à l'«explosion de tous les mondes possibles » (PF 42), ils sentent le sol se dérober peu à peu sous leurs pieds, d'où le fait que Casaubon, Belbo ou Roberto della Griva éprouvent par moments l'impression d'errer dans un espace épistémologique comportant un nombre de dimensions infini, « un labyrinthe [...] qui conduit partout et nulle part » (PF 535), bref, un rhizome deleuzien.

(c) L'imbrication de «territoires » hétéroclites. La confusion sémiotique ainsi pointée par Eco est spectaculairement accrue, dans L'Isola del giorno prima et surtout Baudolino, par les tâtonnements cosmologiques parfois grotesques de ses personnages, qui n'arrivent pas à donner une assise ferme à leurs spéculations (méta-)abductives en s'inscrivant une fois pour toutes dans un "territoire » conceptuel donné. Utilisé ici au sens spécifique que lui attribuent Gilles Deleuze et Félix Guattari dans Mille plateaux, le mot « territoire » désigne, au-delà de sa signification strictement "géographique ", un espace (au sens le plus abstrait du terme) qui «[fait] agencement » (Deleuze et Guattari 1980 : 397), autrement dit un espace qui détermine simultanément les modes d'incarnation des différentes « entités » qui émergent en son sein et l'ensemble de relations qui se développent entre ces entités. Un paradigme scientifique, un genre littéraire, un régime politique, un univers fictionnel, un système social donnés sont autant de «territoires " au sens deleuzien du terme, dans la mesure où ils délimitent un périmètre, un domaine qui rend possible l'actualisation, puis l'identification d'«objets» et de systèmes de rapports spécifiques. Ce qui se passe donc concrètement dans les deux derniers romans de notre 
corpus, c'est que leurs protagonistes se trouvent au cœur d'un enchevêtrement absolument inextricable de «territoires»mutuellement incompatibles, chacun présupposant des modes d'agencement différents des «objets» qui le peuplent: le résultat de cette "confusion des genres", c'est la mise en place d'«assemblages abductifs » constitués à partir d'éléments totalement hétérogènes (cf. fig. 5).

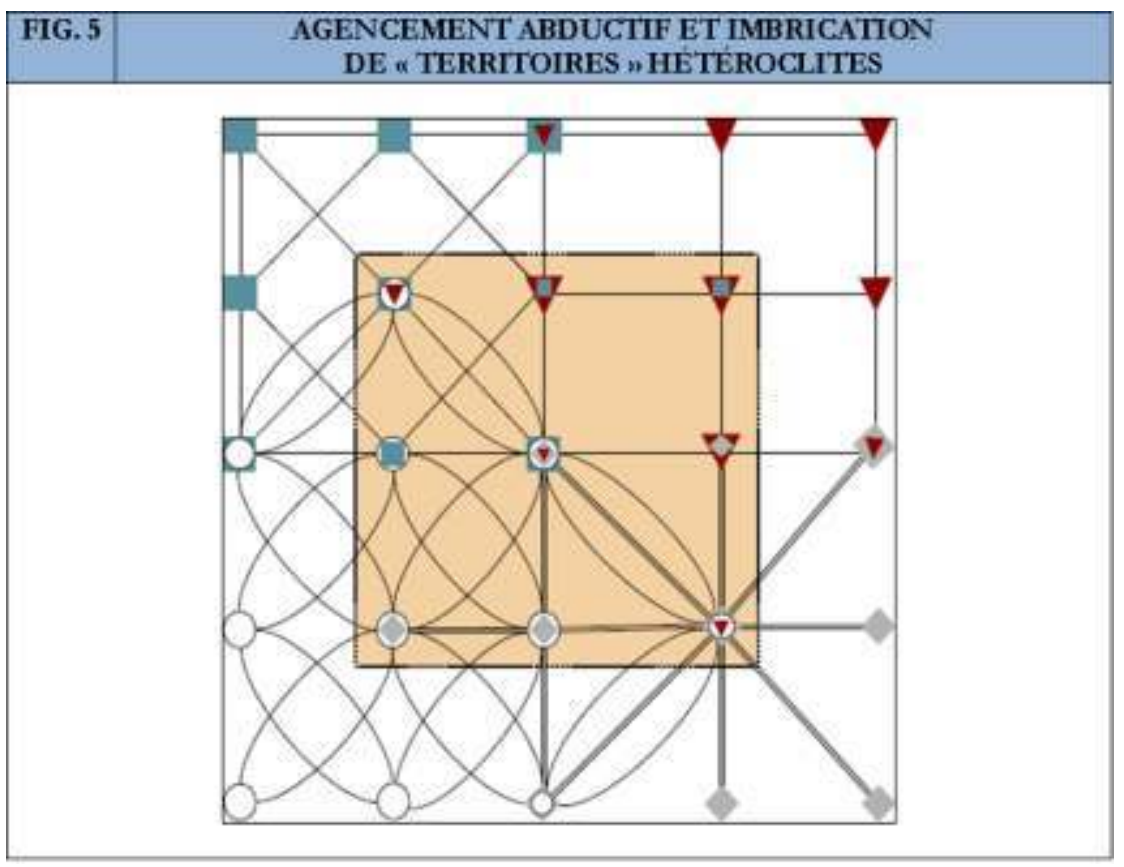

L'exemple qui vient tout de suite à l'esprit, c'est la théorie complètement farfelue inventée par le père Wandendrossel pour expliquer comment Dieu s'y est pris pour recouvrir la Terre d'eau en à peine quarante jours (cf. IGP 259-266) : en tentant de « faire coïncider la dictée de la Bible avec les connaissances que nous avons des lois célestes » ( IGP 254), Wandendrossel propose à Roberto della Griva un mélange effarant de spéculations théologiques, de citations bibliques, de considérations géoastronomiques manifestement absurdes et de raisonnements analogiques, créant ainsi un schéma abductif «hybride» dont le moins qu'on puisse dire est qu'il laisse le lecteur perplexe. Mais le moine Byzantin Zosime, qui explique à Baudolino et ses amis que la Terre a la forme d'un tabernacle, s'avère encore plus inventif que Wandendrossel !

(7) - [...] Je t'ai dit [Zosime s'adresse à Baudolino] que chaque chrétien qui suivrait la vraie foi doit accepter le fait que l'univers monde est fait comme le tabernacle dont parlent les écritures. [...] Dans la partie inférieure du tabernacle il y avait une table avec douze pains et douze fruits, chacun pour un des mois de l'année, tout autour de la table il y avait un socle qui figurait l'Océan, et autour du socle il y avait un cadre large d'un empan qui représentait la terre de l'au-delà, où, à l'orient, se trouve le Paradis terrestre. La voûte représentait le ciel, qui s'appuyait entièrement sur les extrémités de la terre, mais entre la voûte et la base était déployé le voile du firmament au-delà de quoi se trouve le monde céleste que nous ne verrons qu'un jour face à face. En effet, comme a dit Isaïe, Dieu est Celui qui est assis sur la terre, dont les habitants sont comme des locustes, Celui qui, tel un voile ténu, a tendu le ciel et l'a déplié comme une tente. Et le psalmiste loue Celui qui déploie le ciel comme un pavillon. [...]

- Mais tu m'expliques comment était fait le tabernacle, dit Baudolino, pas comment est fait l'univers.

- Mais l'univers est fait comme le tabernacle, et donc si je t'explique comment était le tabernacle, je t'explique comment est l'univers. (B 328) 
Ce qui frappe justement le lecteur en (7), c'est l'hétérogénéité conceptuelle de la description fournie par Zosime: celle-ci relève d'une imbrication de «territoires » (territoire géographique, religieux, épistémologique et stylistique) qui nous paraissent mutuellement incompatibles, dans la mesure où chacun d'entre eux présuppose un « investissement intime de l'espace et du temps » (Zourabichvili 2003:28) et des modes de raisonnement et d'appréhension de l'« objet » radicalement différents.

\section{Conclusion : la quête impossible d'un punto fijo}

27 Les ouvrages fictionnels d'Umberto Eco remettent donc en question de manière très spectaculaire une série de postulats ontologiques et méthodologiques censés garantir le bon fonctionnement du processus (méta-)abductif dans n'importe quel contexte: Eco entend visiblement montrer que ces postulats, hérités pour la plupart du rationalisme philosophique, n'ont pas une validité universelle, ce qui rend ipso facto l'intelligibilité du «réel» aléatoire. L'ambiance d'incertitude qui imprègne les textes de notre corpus découle essentiellement de l'impossibilité de trouver un point de repère stable permettant aux personnages de s'orienter dans le labyrinthe des possibles herméneutiques. La quête de ce punto fijo introuvable se trouve dramatisée au moins dans deux romans :

28 - Dans Il Pendolo di Foucault, du fait que le fameux pendule est censé indiquer «le Point Immobile ", «l'unique point fixe de l'univers» (PF 9), qui se confond aux yeux des occultistes avec l'Umbilicus Telluris. Or, à la fin du roman, le lecteur constate que ce point est occupé par le corps de l'infortuné Jacopo Belbo, qui a été pendu haut et court par le fil $\mathrm{du}$ pendule: ceci est tout sauf un hasard, puisque Belbo incarne justement un agnosticisme ironique typiquement postmoderne qui constitue aux yeux du narrateur la seule attitude existentielle encore concevable dans un monde où le néant est devenu «la Substance du tout » (IGP 475) :

(8) Puis, tandis que l'oscillateur continuait à encourager cette funèbre balançoire, par une atroce combinaison de forces [...], le corps de Belbo était devenu immobile, et le fil avec la sphère se déplaçait comme un pendule de son corps à la terre seulement, le reste - qui reliait Belbo à la voûte - tombant désormais d'aplomb. Ainsi Belbo, réchappé à l'erreur du monde et de ses mouvements, était devenu lui, maintenant, le point de suspension, le Pivot Fixe, le Lieu où se soutient la voûte du monde, et sous ses pieds seulement oscillaient le fil et la sphère, de l'un à l'autre pôle, sans repos, avec la terre qui s'échappait sous eux, montrant toujours un continent nouveau - et la sphère ne savait pas indiquer, et jamais ne le saurait, où se trouvait l'Ombilic du Monde. (PF 606)

29 - Dans L'Isola del giorno prima, du fait que le narrateur ironise sur les désaccords entre les géographes de l'Antiquité et de la Renaissance au sujet de l'emplacement du Premier Méridien, qui sert justement de point de repère permettant de situer tous les autres (cf. IGP 253).

30 L'impossibilité de concevoir des schémas abductifs adossés sur «le roc d'une connaissance certaine» (Heisenberg 1998: 290) entraîne essentiellement trois conséquences :

31 (a) Un affaiblissement des critères permettant de distinguer le "vrai" du "faux", les interprétations pertinentes des interprétations aberrantes. Ces critères ne deviennent pas tout à fait inopérants, mais leur portée se trouve considérablement restreinte : qu'est-ce à 
dire ? Eco montre par exemple, à travers la démarche de Guillaume de Baskerville, que l'efficacité heuristique d'un schéma abductif est optimisée si ce dernier est établi selon un protocole scientifique et étayé par des faits concrets. Or, il est troublant de constater qu'un personnage comme Caspar Wandendrossel tente lui aussi de raisonner comme un " homme de science " (IGP 259) qui fait des calculs "de haute précision » (ibid.) et recueille des données de toutes sortes susceptibles d'étayer ses fumeuses spéculations géo-théologiques en apportant «la preuve de l'experientia » (IGP 266; cf. IGP 301-302 et passim)... Le recours à une forme de « rationalité scientifique » n'est donc pas une garantie méthodologique à toute épreuve, et il en va de même pour les "méthodes de régulation » du processus (méta-)abductif reposant sur le principe ockhamien de l'économie épistémologique: les personnages qui tentent d'appliquer ces méthodes (Léa dans Il Pendolo di Foucault, le moine Byzantin Paphnuce dans Baudolino) disposent en réalité d'un «bon sens" inné qui leur permet d'éliminer intuitivement les constructions interprétatives (jugées) défectueuses et de privilégier les schémas abductifs les plus plausibles (autrement dit, ceux qui permettent d'expliquer le plus de choses avec le moins d'hypothèses initiales). Or, d'où leur vient cette aptitude qui n'est manifestement pas partagée par tout le monde? Comment se fier à un «outil»conceptuel aussi rudimentaire? Le «bon sens » lui-même n'est-il pas déterminé en partie par la doxa qui prévaut dans un paradigme philosophique donné ? Aucune réponse à ces questions n'est fournie dans les romans de notre corpus ...

(b) Une reconfiguration permanente du "réel », dans la mesure où ce dernier n'est pas réductible à un « ordre » universel et immuable. À l'instar de Roberto della Griva contemplant la coupole d'une église romaine, les personnages mis en scène par Eco constatent tous d'une manière ou d'une autre que le monde dans lequel ils évoluent est à la fois instable et fragmenté : son instabilité vient du fait qu'il est soumis au « jeu fluctuant [d']apparences d'ordre qui se réordonn[ent] à chaque nouvelle expérience » (IGP, 504); son morcellement, du fait qu'il est constitué d'une multitude de "territoires » hétéroclites qui «ne se compos[ent] pas en un dessin fini » (ibid.). Les schémas abductifs élaborés dans un tel univers ne peuvent donc avoir qu'une portée locale (cf. Cannon 1989: 13, Musarra-Schroeder 2000 : 488, n. 3) à la fois dans l'espace (plusieurs schémas différents portant sur le même « objet » peuvent coexister simultanément) et dans le temps (chacun de ces schémas peut à tout moment être remis en question).

(c) Une redéfinition du rôle de l'« observateur ", qui ne se contente plus de contempler passivement l'«objet" de référence mais construit activement cet "objet». Il appartient en effet à l'«observateur" d'«assumer ses responsabilités " sur le plan herméneutique et de formaliser à sa guise les "objets complexes » qui s'offrent à son regard, en choisissant délibérément de "mettre en relief» certaines connexions signifiantes au détriment d'autres : « Roberto n'observait pas les constellations : il était condamné à les instituer. Il s'effrayait que l'ensemble se disposât comme une spirale, une coquille d'escargot, un tourbillon » (IGP 503). Une fois évacuée l'erreur consistant à croire que les agencements ainsi mis au jour préexistent nécessairement à l'acte d'observation lui-même, les personnages mis en scène par Eco sont libres de s'adonner au Jeu Suprême de l'interprétation, libres « d'essayer sans cesse de nouvelles lignes de fuite, sans jamais se reposer sur un point central » (IGP 504) : c'est cette exigence intellectuelle permanente, cette liberté de réflexion proprement aérienne, cet émerveillement désabusé qui constituent à nos yeux la quintessence de la « condition postmoderne ». 


\section{Résumés des romans du corpus}

\section{Il Nome della rosa}

En 1327, Guillaume de Baskerville, un moine franciscain d'origine Anglaise, arrive dans une abbaye italienne des Alpes pour participer à une rencontre entre franciscains et dominicains. Guillaume est accompagné d'un novice Allemand, Adso de Melk, qui est le narrateur du roman. Dès son arrivée, il se voit prié par l'abbé d'enquêter sur la mort d'un de ses moines, dont on a retrouvé le cadavre en bas des murailles du monastère. Aidé par son novice, Guillaume se met à l'ouvrage, alors que les morts de moines se succèdent. Très vite, l'enquête est orientée vers les activités de toutes sortes qui ont lieu nuitamment dans la bibliothèque de l'abbaye : le mystère est d'autant plus épais que celle-ci est un vaste labyrinthe théoriquement interdit d'accès à tous les moines excepté l'abbé et le bibliothécaire. Toujours suivi par Adso, Guillaume pénètre secrètement dans le labyrinthe et apprend peu à peu à s'y orienter. Dès lors, les découvertes se succèdent : Guillaume et Adso finissent par comprendre qu'une lutte souterraine est engagée entre plusieurs moines pour récupérer le dernier exemplaire encore existant d'un livre précieux, le deuxième volume de la Poétique d'Aristote. Jugeant le livre impie parce qu'il traite de la comédie, l'ancien bibliothécaire de l'abbaye, Jorge de Burgos, en a empoisonné les pages, de sorte que tous les lecteurs qui essaient de les tourner en les humectant des doigts meurent sur-le-champ. Lors de leur affrontement final avec Jorge, Guillaume et Adso provoquent involontairement un incendie qui détruit l'abbaye de fond en comble. Jorge périt dans l'incendie, non sans avoir auparavant profité de l'occasion pour brûler le livre d'Aristote : son contenu restera donc à jamais inconnu à la postérité. L'enquête étant terminée et le désastre consommé, Guillaume et Adso n'ont plus qu'à reprendre la route.

\section{Pendolo di Foucault}

Trois intellectuels Italiens, Casaubon, Belbo et Diotallevi, essaient de combler leur vide existentiel en menant des recherches sur certaines sectes occultistes, et en particulier les multiples avatars de l'Ordre des Templiers. Invités par l'éditeur qui les emploie à écrire une histoire universelle de l'occultisme, ils se piquent peu à peu au jeu, et décident de forger de toutes pièces, à des fins purement ludiques, une conspiracy theory délibérément extravagante. S'inspirant des spéculations fumeuses d'un certain colonel Ardenti, qui prétend que les Templiers avaient découvert une source de puissance immense, les trois amis postulent que les occultistes de tous bords agissent de concert, en fonction d'un Plan préétabli, pour retrouver la carte indiquant l'emplacement de cette source, ce qui leur permettra de dominer le monde. Mais l'affaire tourne mal ... Leurrés par le scénario ainsi élaboré, les épigones des Templiers se mettent réellement à chercher la carte, dont l'existence ne fait plus de doute à leurs yeux. Belbo commet alors une imprudence fatale : il laisse entendre que ses compagnons et lui ont eu sous les yeux le précieux document - qui indiquerait en fait où se trouve le centre magnétique de la Terre - mais entendent rester discrets sur son contenu. Dès lors, les événements s'accélèrent: piégé par le mystérieux comte d'Agliè, qui se prétend la réincarnation du comte de Saint-Germain, Belbo est enlevé et pendu parce qu'il a refusé (et pour cause) de révéler son secret. Diotallevi meurt d'un cancer et Casaubon, poursuivi par les occultistes, se rend dans la maison de campagne de Belbo et attend stoïquement sa propre fin. 


\section{L'sola del giorno prima} Italien dont le père a été tué lors du siège de Casal. Se sentant trop à l'étroit dans le domaine dont il est désormais le propriétaire, Roberto décide de voyager. Après nombre de pérégrinations, il se rend à Paris où il fréquente $\mathrm{M}^{\mathrm{me}}$ de Rambouillet et se familiarise avec des mouvements culturels comme le libertinage, le baroque et la préciosité. Toutefois, à la suite d'un malentendu, il est arrêté par le cardinal Mazarin, qui lui confie une mission de la plus haute importance: il doit embarquer incognito dans la flûte Amaryllis pour espionner un Anglais nommé d'Igby, qui aurait découvert une méthode révolutionnaire permettant aux marins de déterminer avec une marge d'erreur négligeable la longitude où ils se trouvent. Mais l'Amaryllis fait naufrage dans un archipel du Pacifique et tous ses passagers périssent, à l'exception de Roberto. Épuisé, ce dernier finit par se réfugier dans une autre flûte, le Daphné: il y rencontre un jésuite, le père Caspar Wandendrossel, unique survivant d'un équipage exterminé par des cannibales. Wandendrossel prétend que le Daphné se trouve immobilisé juste en-deçà du 180 ème méridien, au large d'une île qui, elle se trouverait juste au-delà : il en déduit que, si lui et Roberto pouvaient débarquer sur l'île, ils reviendraient un jour en arrière... Cette hypothèse reste toutefois invérifiable, puisque les deux personnages ne savent pas nager et n'ont pas de barque à leur disposition. Le jésuite se noie finalement en essayant de marcher au fond de la mer abrité sous une cloche de son invention, et Roberto, qui tente malgré tout de rejoindre « l'île du jour d'avant » à la nage, est emporté par les courants et disparaît.

\section{Baudolino}

Baudolino, un Italien de basse extraction élevé aux plus hautes fonctions par l'empereur Allemand Frédéric Barberousse, rencontre en 1204 à Constantinople Nikitas Khoniatis, un haut dignitaire Byzantin à qui il raconte l'histoire de sa vie. Issu d'une famille de paysans Frioulans, Baudolino devient le fils adoptif de Barberousse. À seize ans, on l'envoie faire des études à Paris, où il rencontre d'autres personnages (Boron, Kyot, Abdul, le Poète...) qui deviendront ses amis. Avec eux, il est chargé d'accompagner l'Empereur en Asie Mineure lors de la Troisième Croisade. Mais Barberousse est tué en 1190 dans le château d'Ardzrouni, un noble d'origine arménienne. Baudolino et ses compagnons partent alors vers l'Est, à la poursuite d'un moine félon nommé Zosime : celui-ci a été en possession d'une carte censée indiquer l'emplacement exact d'un royaume semi-mythique, la terre du Prêtre Jean. Baudolino et ses compagnons, qui ont eux-mêmes rédigé à Paris un des récits censés confirmer l'existence du royaume de Jean, entendent trouver l'emplacement exact de cette contrée lointaine. Après force pérégrinations, ils y parviennent : la terre de Jean s'avère un pays étrange, peuplé de monstres de toutes sortes et gouverné par le fils du Prêtre, le Diacre Jean. Malgré leurs efforts, Baudolino et ses amis ne pourront jamais rencontrer le Prêtre lui-même. Ils en sont à se demander s'il existe réellement, quand le royaume de son fils est envahi par les Huns qui détruisent sa capitale, Pndapetzim. Nos héros n'ont d'autre choix que de prendre la fuite, et, après de nouvelles péripéties, les survivants du groupe - dont Baudolino - se retrouvent à Constantinople au moment de sa prise par les Croisés en 1204. La boucle est bouclée et le récit de Baudolino terminé. Dans l'avant-dernier chapitre du livre, le protagoniste décide finalement de quitter 
Constantinople et de repartir vers le royaume du Prêtre Jean, dont l'existence ne fait désormais aucun doute pour lui. Nikitas est attristé par son départ, mais reste dubitatif quant à la véracité de son récit.

\section{BIBLIOGRAPHIE}

Michel Bitbol (1996) : Mécanique quantique. Une introduction philosophique, Paris, Flammarion, coll. " Nouvelle Bibliothèque Scientifique ».

JoAnn Cannon (1989) : Postmodern Italian Fiction : the crisis of reason in Calvino, Eco, Sciascia, Malerba, Rutherford, Fairleigh Dickinson University Press.

Catherine Chevalley (1995) : « Nature et loi dans la philosophie moderne », in Notions de philosophie, Denis Kambouchner éd., Paris, Gallimard, coll. « Folio Essais », pp. 127-230.

Gilles Deleuze (1968) : Différence et répétition, Paris, Presses Universitaires de France, coll. «Épiméthée ».

(1969) : Logique du sens, Paris, Minuit, coll. « Critique ».

Gilles Deleuze et Félix Guattari (1980) : Capitalisme et schizophrénie, tome 2, Mille plateaux, Paris, Minuit, coll. «Critique ».

Bernard d'Espagnat (1985) : Une Incertaine réalité. Le monde quantique, la connaissance et la durée, Paris, Gauthier-Villars.

Bernard d'Espagnat (1994) : Le Réel Voilé. Analyse des concepts quantiques, Paris, Fayard, coll. « Le Temps des sciences $»$.

Umberto Eco (1962) : Opera aperta. Forma e indeterminazione nelle poetiche contemporanee, Milan, Bompiani.

Umberto Eco (1976 [ $\left.\left.{ }^{1} 1975\right]\right)$ : A Theory of semiotics, Bloomington/Londres, Indiana University Press, coll. «Advances in semiotics ».

Umberto Eco (1982 [11980]) : Le Nom de la rose [NR], trad. de l'italien par Jean-Noël Schifano, Paris, Grasset/Fasquelle, coll. « Le Livre de Poche ».

Umberto Eco (1985 [11983]) : Apostille au Nom de la rose, trad. de de l'italien par Myriem Bouzaher, Paris, Grasset, coll. « Le Livre de Poche/Biblio Essais ».

Umberto Eco (1990 [11988]) : Le Pendule de Foucault [PF], trad. de l'italien par Jean-Noël Schifano, Paris, Grasset/Fasquelle, coll. « Le Livre de Poche ».

Umberto Eco (1992 [11990]) : Les Limites de l'interprétation, trad. de l'italien par Myriem Bouzaher, Paris, Grasset/Fasquelle, coll. « Le Livre de Poche/Biblio Essais ».

Umberto Eco (1996 [ $\left.\left.{ }^{1} 1994\right]\right):$ L'Île du jour d'avant [IGP], trad. de l'italien par Jean-Noël Schifano, Paris, Grasset/Fasquelle, coll. « Le Livre de Poche ».

Umberto Eco (1999 [11997]) : Kant et l'ornithorynque, trad. de l'italien par Julien Gayrard, Paris, Grasset/Fasquelle. 
Umberto Eco (2002 [12000]) : Baudolino [B], trad. de l'italien par Jean-Noël Schifano, Paris, Grasset/ Fasquelle, coll. « Le Livre de Poche ».

Cristina Farronato (1999) : " The Theory of abduction and The Name of the Rose », in Semiotics 1998, C. W. Spinks et John Deely éds, Bern, Peter Lang Publishing, pp. 71-81.

Cristina Farronato (2003) : Eco's chaosmos : from the Middle Ages to postmodernity, Toronto, University of Toronto Press.

Christophe Gelly (2005) : Le Chien des Baskerville. Poétique du roman policier chez Conan Doyle, Lyon, Presses Universitaires de Lyon, coll. « Champ Anglophone ».

Werner Heisenberg (1998) : Philosophie. Le manuscrit de 1942, trad. de l'allemand par Catherine Chevalley, Paris, Seuil, coll. « Sources du savoir ».

Linda Hutcheon (1988) : A Poetics of postmodernism : history, theory, fiction, New York/Londres, Routledge.

Jean-Marie Klinkenberg (1996) : Précis de sémiotique générale, Paris, Seuil/De Boeck, coll. « Points Essais ».

Thomas S. Kuhn (1962) : The Structure of scientific revolutions, Chicago, The University of Chicago Press.

Jean-François Lyotard (1979) : La Condition postmoderne : rapport sur le savoir, Paris, Minuit, coll. « Critique ».

Brian McHale (1987) : Postmodernist fiction, New York/Londres, Methuen, coll. « University Paperbacks ».

Ulla Musarra-Schroeder (2000) : «Mondes possibles et encyclopédie : stratégies narratives dans les romans d'Umberto Eco ", in Au Nom du sens : autour de l'œuvre d'Umberto Eco, Jean Petitot et Paolo Fabbri éds, Paris, Grasset, pp. 485-502.

Stefano Tani (1984): The Doomed detective : the contribution of the detective novel to postmodern American and Italian fiction, Carbondale/Edwardsville, Southern Illinois University Press, coll. « Literary structures ».

Ludwig Wittgenstein (1993) : Tractatus logico-philosophicus, suivi de Investigations philosophiques, trad. de l'allemand par Pierre Klossowski, Paris, Gallimard, coll. « Tel ».

Ilias Yocaris (2006a) : « Signes et interprétation des signes dans la fiction postmoderne : le schéma de l'enquête dans Le Nom de la rose ", in Traces, énigmes, problèmes : émergence et construction du sens, Jerzy Karczmarczuk éd., Paris, ENST, pp. 197-208.

Ilias Yocaris (2006b) : « Nomadisme conceptuel, nomadisme spatial : le thème de la "déterritorialisation" dans Baudolino d'Umberto Eco ", Cadernos de Literatura Comparada, 14-15, Textos e mundos em deslocacão, tome 2, pp. 93-112.

François Zourabichvili (2003) : Le Vocabulaire de Deleuze, Paris, Ellipses, coll. « $\varphi$ / Vocabulaire de... ».

\section{NOTES}

1. D'où le fait que des enquêteurs comme Dupin (dans les nouvelles policières de Poe) ou Sherlock Holmes (dans les romans de Conan Doyle) ne se trompent jamais dans leurs «hypothèses de travail » : cf. Eco 1992 : 281-283. 
2. Un schéma narratif de ce genre, qui rappelle étrangement certaines interprétations de la mécanique quantique datant des années 1920 (cf. Bitbol 1996 : 246-247, Yocaris 2006 : 203, n. 8), apparait (tel quel ou avec des variantes) dans bon nombre d'œuvres de fiction postmodernes retraçant le déroulement d'une enquête, comme Les Gommes d'Alain Robbe-Grillet (1953) ou The Element of crime de Lars Von Trier (1984) : dans le film de Von Trier comme dans le roman de Robbe-Grillet, la « ligne de démarcation » entre « sujet observant » et « objet observé » disparaît, puisque l'enquêteur finit par commettre lui-même le crime qu'il était chargé d'élucider (dans Les Gommes) ou de prévenir (dans The Element of crime)...

3. Au vu du déroulement de l'intrigue, une telle conclusion pourrait sembler trop radicale : après tout, la démarche (méta-)abductive de Guillaume n'est pas un échec intégral, puisqu'il a eu raison de supposer que les morts sur lesquelles il enquête découlent toutes (directement ou indirectement) de la lutte pour la possession d'un livre (correctement identifié comme étant le deuxième volume de la Poétique) aux pages empoisonnées. Si Guillaume éprouve un sentiment de frustration cognitive, ce n'est pas parce qu'il est submergé par le désordre, mais parce qu'il ne réussit à imposer au réel qu'un ordre partiel : il a bien repéré la «cause première » qui est à l'origine de toutes les morts, mais il n'a pas compris que celles-ci se sont enchaînées de manière plus ou moins fortuite, et non point comme des manifestations nécessaires d'un Grand Dessein initial, analogue à la Providence Divine [cf. NR 475].

4. Cet a priori méthodologique se trouve exprimé sur le plan stylistique par l'emploi systématique de l'uniception, qui permet à Legrand d'évacuer de son discours (et, par extension, du «monde possible » au sein duquel il évolue) toute forme d'incertitude : "The "good glass", I knew, could have reference to nothing but a telescope "; "Of course, the "forty-one degrees and thirteen minutes" could allude to nothing but elevation above the visible horizon », «[T]he phrase "main branch, seventh limb, east side", could refer only to the position of the skull upon the tree ", etc.

\section{AUTEUR}

\section{ILIAS YOCARIS}

IUFM de Nice et Université de Nice-Sophia Antipolis (CTEL, CIRCPLES) 